\title{
PARAMETER STABILITY MARGINS FOR POLYNOMIAL UNCERTAINTY STRUCTURES: A POLYNOMIAL PROGRAMMING APPROACH
}

\author{
Mohammad Bozorg ${ }^{\text {a }}$, Hanif D. Sherali ${ }^{\text {b }}$, Edward J. Davison ${ }^{c}$ \& Ali Delavar-Khalafi ${ }^{\text {d }}$ \\ ${ }^{a}$ Dept. of Mechanical Eng., University of Yazd, P.O. Box 89195-741, Yazd, Iran \\ ${ }^{b}$ Grado Dept. of Industrial and Systems Eng., Virginia Polytechnic Institute \\ and State University, Blacksburg, VA, USA \\ ${ }^{c}$ Systems Control Group, Dept. of Electrical and Computer Eng., \\ University of Toronto, Toronto, ON, Canada \\ ${ }^{d}$ Dept. of Mathematics, Shahrekord University, Shahrekord, Iran
}

\begin{abstract}
In this paper, LTI systems with parameter uncertainty are considered. For such systems, a method is presented for determining permissible perturbations of the parameters of the system that maintain stability (the so-called parameter stability margins) for the case in which the coefficients of the characteristic equation of the system are polynomial functions of the uncertain parameters. A globally convergent polynomial programming technique is used to solve the underlying parametric nonconvex subproblems for computing the stability margins. The approach is illustrated using a four-parameter numerical example. Copyright (C) 2005 IFAC.
\end{abstract}

Keywords: Robust stability, Uncertain linear systems, Uncertain polynomials, Global optimization, Polynomial methods.

\section{INTRODUCTION}

Control of linear systems with uncertain physical parameters has been a main subject of research in the field of control engineering over the last two decades (Barmish, 1994; Bhattacharyya, et al., 1995; Ackermann, 2002). One approach to deal with this problem is to study the behavior of the characteristic equations of these systems. In particular, the effects of uncertain parameters on the location of the roots of the polynomials, and thence on the stability and the performance of these systems, has been widely investigated.

For the cases when the coefficients of the polynomials are linear functions of the system's uncertain parameters, many powerful tools have been developed (see, e.g., Barmish, 1994; Ackermann, 2002). However, in many engineering applications, the coefficients often turn out to be multilinear, polynomial or nonlinear functions of the system's uncertain parameters (Bhattacharyya, et al., 1995; Ackermann, 2002), and in these cases, the research has been less fruitful. Several representative contributions in these problem areas, mainly in the stability analysis context, are given in (Chapellat, et al., 1993; Djaferis, 1995; Polyak and J. Kogan, 1995; Zettler and Garloff, 1998).

A fundamental problem in robust control is to compute the stability margins for parameter perturbations, i.e., to determine the maximum allowable perturbations in the uncertain parameters of a stable system without losing stability. Here, stability is defined with respect to an arbitrary region $D$ in the complex plane, and is called " $D$-stability", and such stability margin results are used in the design of robust controllers (Ackermann, 2002; Bozorg and Nebot, 1999). For the case of linear dependency of the coefficients with respect to uncertain parameters, several algorithms have been developed for determining the $D$-stability margins (Hinrichsen and Pritchard, 1989; Qiu and Davison, 
1989; Tsypkin and Polyak, 1991). However, for the cases when the coefficients are polynomial functions of uncertain parameters, few results are available to calculate the system's stability margins. In (Ackermann, 2002; Ackermann, et al., 1990), a graphical method is presented to visualize the stability domains of polynomials in the parameter space and this method is suitable for situations when the number of uncertain parameters is small. In (Sideris and Sánchez Peña, 1989), it is shown that a polynomial with a polynomial uncertainty structure and a polytopic domain of parameter uncertainty, can be transformed into a polynomial with a multilinear uncertainty structure in conjunction with a new polytopic uncertainty domain. Thus, multilinear results can be obtained in these cases. For the case of multilinear dependency, the well-known Mapping Theorem (Zadeh and Desoer, 1963) is one of the few tools available for checking robust stability; however, it is recognized that the sufficiency conditions of this theorem can lead to conservative results (Polyak and Kogan, 1995). The Mapping Theorem is used in (Sideris and Sánchez Peña, 1989; Sideris and Sánchez Peña, 1989; De Gaston and Safonov, 1988; Keel and Bhattacharyya, 1993) to develop several algorithms for determining lower bounds for stability margins for the multilinear case. For the cases of multilinear and polynomial uncertainty structures, the problem of computing $D$ stability margins has remained an open problem.

In this paper, an algorithm is presented for the computation of stability margins for the case of polynomial uncertainty structures. Polynomial programming results are exploited for computing the stability margins. The zero exclusion results (see, e.g., Barmish, 1994) are used to formulate the stability margin computation as a polynomial optimization problem. An algorithm is then presented to compute the $D$-stability margins via the solution of a parametrized family of polynomial programming problems using the method described in (Sherali and Tuncbilek, 1992, 1997)]. It is also shown how the structure of the objective function and the physical limits of parameter variations can be used to tighten the lower bounds and to facilitate the effective application of this methodology. A numerical example is also provided to illustrate the proposed approach.

\section{PRELIMINARIES}

Consider the polynomial

$$
Q(q, s)=a_{n}(q) s^{n}+\cdots+a_{1}(q) s+a_{0}(q), a_{n}(q) \neq 0,(1)
$$

where the coefficients $a_{n}(q), \ldots, a_{1}(q), a_{0}(q)$ are polynomial functions of the parameters $q=\left[q_{1}, q_{2}, \ldots, q_{m}\right]^{\mathbf{T}} \in \mathbf{R}^{\mathbf{m}}$.

Let $D$ be an open subset of the complex plane and denote its contour by $C_{D}$. We cite the following wellknown theorem on the robust stability of linear systems.

Theorem 1. (Zero Exclusion Theorem) (See Barmish, 1994): Consider the family of invariant-degree polynomials (1) with $q \in \mathbf{Q}$, where $\mathbf{Q}$ is an uncertainty set that is path-wise connected, which has at least one $D$-stable member. Then, the family of polynomials is $D$-stable if and only if

$$
0 \notin Q(q, u), \forall q \in \mathbf{Q}, \forall u \in C_{D} .
$$

To check the necessary and sufficient conditions of Theorem 1 for $D$-stability, the contour of the region must be swept. A point $u$ on the contour $C_{D}$ can always be expressed as a function of a sweeping variable $z$, i.e., $u=u(z), u \in C_{D} \subset \mathbf{C}, z \in \mathbf{Z} \subset \mathbf{R}$; for instance, the contour of the unit circle can be represented by $u(z)=e^{j z}, z \in[0,2 \pi]$, where $j=\sqrt{-1}$. The substitution of $s=u(z)$ in (1) results in

$$
\begin{aligned}
& Q(q, u(z))=Q_{R}(q, z)+j Q_{I}(q, z), \\
& Q_{R}(q, z):=\operatorname{Re}[Q(q, u(z))]=\sum_{i=0}^{n} \alpha_{i}(z) a_{i}(q), \\
& Q_{I}(q, z):=\operatorname{Im}[Q(q, u(z))]=\sum_{i=0}^{n} \beta_{i}(z) a_{i}(q) \\
& \alpha_{i}(z):=\operatorname{Re}[u(z)]^{i}, \beta_{i}(z):=\operatorname{Im}[u(z)]^{i}, i=0, \ldots, n .
\end{aligned}
$$

Define the weighted $l_{2}$-distance (norm) of two arbitrary points $q, q^{\prime} \in \mathbf{R}^{m}$ in the parameter space as

$$
\delta\left(q, q^{\prime}\right):=\left[\sum_{k=1}^{m}\left(\left(q_{k}-q_{k}^{\prime}\right) / w_{k}\right)^{2}\right]^{1 / 2},
$$

where $w_{k}>0, k=1, \ldots, m$, are weights.

Consider the family of polynomials

$$
\mathrm{P}:=\{Q(q, s): q \in B(\bar{q}, b)\},
$$

where the parameter uncertainty domain given by

$$
B(\bar{q}, b):=\{\widetilde{q}: \delta(\bar{q}, \widetilde{q})<b\},
$$

is a hypersphere in the parameter space, $b$ is the size of the hypersphere, and $\bar{q}$ is the center of the hypersphere (corresponding to the nominal value of the parameter vector). The family is assumed to be $D$-stable at $q=\bar{q}$.

\section{STABILITY MARGINS}

In this section, the problem of calculating the $D$ stability margins is addressed. In other words, the maximum size $(b)$ of the parameter hypersphere (8) to preserve $D$-stability is determined.

To calculate the $D$-stability margins, we define the following optimization problem (Tesi and Vicino 1990; Desages, et al., 1991): given $\delta\left(q, q^{\prime}\right)$ defined in (6), $Q_{R}$ and $Q_{I}$ defined in (4) and (5), respectively, 
and the nominal value of $q=\bar{q}$, let the sweeping variable $z \in \mathbf{Z}$ be fixed and define

$$
\rho(z):=\min _{q}\left\{\delta(\bar{q}, q): Q_{R}(q, z)=0, Q_{I}(q, z)=0\right\}
$$

Let the function $\rho(z)$ be called the Minimum Distance Function (MDF).

A necessary condition of Theorem 1 for $D$-stability, is the invariance of the degree of the perturbed polynomials. This implies that the parameter perturbations must not result in the nullification of $a_{n}(q)$. To satisfy this condition, the perturbation $\delta(\bar{q}, \widetilde{q})$, where $\widetilde{q}$ represents a perturbed value of $q$, must be less than the following optimal value:

$$
\eta:=\min _{q}\left\{\delta(\bar{q}, q): a_{n}(q)=0\right\} \text {. }
$$

Note that the optimization (10) is independent of the sweeping variable. Thence, it is not required to search the contour to evaluate $\eta$. The following theorem provides a characterization for the size of uncertainty hypersphere (8) for $D$-stability.

Theorem 2 . The family (7) is $D$-stable if and only if

$$
b<\gamma,
$$

where

$$
\gamma:=\min \left\{\eta, \min _{z} \rho(z): z \in \mathbf{Z}\right\} .
$$

Proof. A member of the family (7) is stable at $q=\bar{q}$ by assumption. If the size of the perturbation hypersphere $b$ is smaller than $\eta$, the condition $a_{n} \neq 0$ is satisfied for any $\tilde{q} \in B(\bar{q}, b)$; therefore, the preconditions of Theorem 1 are met. If the size of the perturbation hypersphere $b$ is smaller than $\min _{z \in \mathbf{Z}} \rho(z)$, this implies that for any $\widetilde{q} \in B(\bar{q}, b)$ and $z \in \mathbf{Z}$, the constraints of (9) will not be satisfied, and so, the necessary and sufficient conditions of Theorem 1 for $D$-stability are met. This completes the proof.

Remark 1. For any $z$, if (9) is infeasible, then evidently $\rho(z)=\infty$. Else, given a feasible solution $\hat{q}$ to (9), the problem is equivalent to one that further restricts $\delta(\bar{q}, q) \leq \delta(\bar{q}, \hat{q})$. Then, because this amounts to minimizing a continuous function over a nonempty set, by Weierstrass' Theorem (see e.g., Rudin, 1976), a minimum exists that defines $\rho(z)$.

\section{PROPOSED OPTIMIZATION APPROACH}

In this section, an algorithm is presented for solving (9) in order to compute MDFs. A similar procedure can be used for solving (10). For given values of the sweeping parameter $z$ and the nominal parameter vector $\bar{q}$, the following Minimum Distance Estimation (MDE) problem is defined:
Minimize $f(q):=(\delta(\bar{q}, q))^{2}=\sum_{k=1}^{m} c_{k}\left(q_{k}-\bar{q}_{k}\right)^{2}$

subject to:

$$
\begin{aligned}
& Q_{R}(q, z)=\sum_{i=0}^{n} \alpha_{i}(z) a_{i}(q)=0, \\
& Q_{I}(q, z)=\sum_{i=0}^{n} \beta_{i}(z) a_{i}(q)=0,
\end{aligned}
$$

where $c_{k}=1 / w_{k}^{2}, \forall k=1, \ldots, m$. Denote the highest order of any polynomial term in $q$ within the constraints (13b) and (13c) by $\Delta, \Delta \geq 2$.

\section{Optimization Algorithm}

We will now describe an algorithm to solve problem (13). The following steps are adopted in solving the MDE problem:

Step 1. (Heuristic Solution). Assume that by starting at some suitable solution (perhaps $q=\bar{q}$ or some known unstable (feasible) solution). A local search procedure is applied to find an approximate (perhaps locally minimizing) solution $\hat{q}$, having an objective value $\hat{v}$.

Step 2. (Deriving Bounds on q). Based on the solution obtained at Step 1, we can impose the objective cut:

$$
f(q) \leq \hat{v} .
$$

By the nature of $f(q)$, which is comprised of nonnegative, separable terms, it is asserted that

$$
c_{k}\left(q_{k}-\bar{q}_{k}\right)^{2} \leq \hat{v}, \forall k=1, \ldots, m,
$$

which, in turn, permits us to derive lower and upper bounds on the $q$-variables:

$$
q_{k l} \leq q_{k} \leq q_{k u}, \forall k=1, \ldots, m,
$$

where

$$
q_{k \ell}=\bar{q}_{k}-\left(\hat{v} / c_{k}\right)^{1 / 2}, q_{k u}=\bar{q}_{k}+\left(\hat{v} / c_{k}\right)^{1 / 2} .
$$

Remark 2. The purpose of finding a good quality feasible solution $\hat{v}$ at Step 1 is to permit the derivation of tight bounds (16) at Step 2. This can significantly enhance the convergence performance of the algorithm. A sequential linear programming methodology is also described in (Sherali and Tuncbilek, 1997) to tighten the derived bounds (16) while retaining optimality for the underlying polynomial program (9). This would be highly beneficial to utilize, as supported by the computational results reported in (Sherali and Tuncbilek, 1997).

Step 3. (Generation of Bound-Factor Constraints). Let $\bar{M}$ denote the set comprised of $\Delta$ replicates of each of the indices $1, \ldots, m$. Then, the set of boundfactor constraints as defined in (Sherali and Tuncbilek, 1992) are given by: 


$$
\begin{aligned}
& \prod_{k \in K_{1}}\left(q_{k}-q_{k \ell}\right) \prod_{k \in K_{2}}\left(q_{k u}-q_{k}\right) \geq 0, \\
& \forall K_{1} \subseteq \bar{M}, \forall K_{2} \subseteq \bar{M}
\end{aligned}
$$

with $\left|K_{1}\right|+\left|K_{2}\right|=\Delta$. Note that there are

$$
\sum_{r=0}^{\Delta}\left(\begin{array}{c}
m+r-1 \\
r
\end{array}\right)\left(\begin{array}{c}
m+(\Delta-r)-1 \\
(\Delta-r)
\end{array}\right)
$$

such bound-factor constraints.

Step 4. (Defining a Linearization under the RLT Substitution). Define the RLT product variables

$$
Q_{K}:=\underset{k \in K}{\pi} q_{k}, \forall K \subseteq \bar{M}, 2 \leq|K| \leq \Delta,
$$

where the indices in $K$ are assumed to be ordered in non-decreasing sequence. Note that there are $\left(\begin{array}{c}m+\Delta \\ \Delta\end{array}\right)-(m+1)$ such distinct $Q$-variables. For convenience, we also let $Q_{k}:=q_{k}, \forall k=1, \ldots, m$ and $Q_{\{\varnothing\}}=1$. Furthermore, for any polynomial function $h(q)$, let $[h(q)]_{L}$ denote the linearized function obtained in variables $q$ and $Q$, upon substituting (18) for each distinct polynomial product term. Accordingly, let us define:

$$
\Omega=\left\{q: q_{k \ell} \leq q_{k} \leq q_{k u}, k=1, \ldots, m\right\},
$$

and recognizing that the Constraints (17) are contingent upon these bounds, let us define

$$
\begin{aligned}
& X_{L}(\Omega)=\left\{(q, Q): \sum_{i=0}^{n} \alpha_{i}(z)\left[a_{i}(q)\right]_{L}=0,\right. \\
& \sum_{i=0}^{n} \beta_{i}(z)\left[a_{i}(q)\right]_{L}=0, \\
& {\left[\prod_{k \in K_{1}}\left(q_{k}-q_{k \ell}\right) \prod_{k \in K_{2}}\left(q_{k u}-q_{k}\right)\right]_{L} \geq 0,} \\
& \left.\forall K_{1} \subseteq \bar{M}, \forall K_{2} \subseteq \bar{M} \text { with }\left|K_{1}\right|+\left|K_{2}\right|=\Delta, q \in \Omega\right\} .
\end{aligned}
$$

Step 5. (Optional Tightening of Bounds in $\Omega$ ). Note that $f(q)$ defined in $(13 \mathrm{a})$ is a quadratic function, and that we can impose (14) as an additional constraint in the problem. Accordingly, define

$$
X_{L}(\Omega, \hat{v}) \equiv X_{L}(\Omega) \cap\left\{(q, Q):[f(q)]_{L} \leq \hat{v}\right\} .
$$

In lieu of deriving the bounds (16) based solely on (14), we can now sequentially minimize and maximize each $q_{k}$ in turn, subject to the linear constraints (20), in order to possibly tighten the lower and upper bounds on $q_{k}$, for each $k=1, \ldots, m$. Each time a bound is actually improved, we can update $\Omega$ and the corresponding set $X_{L}(\Omega)$ used in (20) for the next such problem. Having performed one complete pass through the variables $k=1, \ldots, m$, in case the volume of the resulting $\Omega$ is less than an arbitrary percent (e.g., 90\%) of the original $\Omega$, we can repeat this step. Note that these LPs are typically easily solved, and frequently provide a beneficial tightening in bounds.
Step 6. (Branch-and-Bound Procedure). Given any $\Omega$, the prescribed branch-and-bound procedure presented in (Sherali and Tuncbilek, 1992) for solving MDE uses the following linear program to compute lower bounds:

$$
L P(\Omega): \underset{(q, Q)}{\operatorname{Minimize}}\left\{[f(q)]_{L}:(q, Q) \in X_{L}(\Omega)\right\} .
$$

Step 6a. (Initialization). Let $q^{*}=\hat{q}$ be the incumbent solution of objective value $v^{*}=\hat{v}$. (If no feasible solution is available, let $q^{*}$ be null with $v^{*}=\infty$.) Set the iteration counter $r=1$, and let the set of active nodes be $T_{r}=\{1\}$, with $t(r)=1$, and $\Omega^{1} \equiv \Omega$. Solve $\operatorname{LP}(\Omega)$ and let $(\tilde{q}, \tilde{Q})$ be the solution obtained with objective value $L B_{1}$. If $\tilde{q}$ is feasible to MDE, update $q^{*}$ and $v^{*}$, if necessary, and if $\left(L B_{1}+\varepsilon\right) \geq v^{*}$ for some optimality tolerance $\varepsilon \geq 0$, then stop; $q^{*}$ is an $\varepsilon$-optimum for MDE. Otherwise, select a branching index $w \in\{1, \ldots, m\}$ as follows. First, find $K^{*} \subseteq \bar{M}$ for which

$$
K^{*} \in \underset{\substack{K \subseteq \bar{M} \\ 2 \leq|K| \leq \Delta}}{\arg \max }\left|\widetilde{Q}_{K}-\underset{k \in K}{\pi} \widetilde{q}_{k}\right| .
$$

Then, for each $k \in K^{*}$, let $n_{k}$ be the number of times $k$ appears in $K^{*}$, and accordingly, select

$$
w \in \underset{k \in K^{*}}{\arg \max }\left|\widetilde{Q}_{K^{*}}-\widetilde{q}_{k}^{n_{k}} \widetilde{Q}_{K^{*}-\left\{n_{k} \text { occurances of } k\right\}}\right| .
$$

Proceed to Step $6 b$.

Step 6b. (Partitioning Step). Partition the selected active node $\Omega$ into two sub-hyperrectangles by splitting the current bounds on $q_{w}$ at the value $\tilde{q}_{w}$. Update $T_{r}$ by adding these two children nodes (indexed $t_{1}$ and $t_{2}$ ) and removing the parent node $t(r)$.

Step 6c. (Bounding Step). Solve $\operatorname{LP}(\Omega)$ for $\Omega$ corresponding to each of the two new nodes $t_{1}$ and $t_{2}$ generated, in order to derive respective lower bounds $\mathrm{LB}_{t_{1}}$ and $\mathrm{LB}_{t_{2}}$. Update the incumbent solution if possible, and determine a branching variable for each node as necessary, using the process described in Step 6a.

Step 6d. (Fathoming Step). Update $T_{r+1}=T_{r}-$ $\left\{t \in T_{r}:\left(L B_{t}+\varepsilon\right) \geq v^{*}\right\}$. If $T_{r+1}=\varnothing$, then stop; the incumbent solution is $(\varepsilon-)$ optimal. Otherwise, increment $r$ by one and proceed to Step 6(e).

Step 6e. (Node Selection Step). Select an active node $t(r) \in \arg \min \left\{L B_{t}: t \in T_{r}\right\}$, and return to Step $6 \mathrm{~b}$.

The convergence proofs in (Sherali and Tuncbilek, 1992) establish that this branch-and-bound methodology will either finitely determine an optimal solution, or else, will generate an infinite sequence of lower bounding solutions, any accumulation point of which would be an optimal solution to MDE. Of course, in the latter case, in practice, once the global 
lower and upper obtained on Problem MDE reach some desired tolerance $\varepsilon>0$, which must occur in finite number of steps, we can terminate the procedure.

Remark 3. Observe that the purpose of solving MDE is to determine $\rho(z)$ as described by (9) so that by examining a plot of $\rho(z)$ as a function of the sweeping variable $z$, we can determine the stability margin $\gamma$ from (12). Alternatively, by letting $z$ itself be a variable in MDE, we can directly compute

$$
\min \{\rho(z): z \in \mathbf{Z}\}
$$

by solving a single polynomial program using the foregoing approach, although this problem would be one of a higher order and hence, more complex to solve.

\section{NUMERICAL EXAMPLE}

The following example is included to illustrate the application of the proposed algorithm. Consider the feedback control system of Fig. 1, which corresponds to Example 5.1 of (Bhattacharyya, et al., 1995), where

$$
\begin{aligned}
& F(s)=\frac{s+1}{s+2}, P(s)=\frac{s+q_{1}}{s^{2}+q_{2} s+4}, \\
& Q(s)=\frac{s+q_{3}}{s^{3}+3 s^{2}+q_{4} s+0.1} .
\end{aligned}
$$

In this case, the parameter vector given by $q=\left[q_{1}, q_{2}, q_{3}, q_{4}\right]^{\mathrm{T}}$ is assumed to be subject to uncertainty, and its nominal value is assumed to be $\bar{q}=[3,2,5,2]^{\mathrm{T}}$. The characteristic equation of the system is obtained as

$$
\begin{aligned}
& Q(q, s)=s^{6}+\left(q_{2}+5\right) s^{5} \\
& +\left(5 q_{2}+q_{4}+10\right) s^{4}+\left(q_{2} q_{4}+6 q_{2}+2 q_{4}+21.1\right) s^{3} \\
& +\left(2 q_{2} q_{4}+q_{1}+0.1 q_{2}+q_{3}+4 q_{4}+25.2\right) s^{2} \\
& +\left(q_{1} q_{3}+q_{1}+0.2 q_{2}+q_{3}+8 q_{4}+0.4\right) s+\left(q_{1} q_{3}+0.8\right),
\end{aligned}
$$

which is a polynomial whose coefficients are multilinear functions of the parameters of the system. This is a special case of polynomial dependency in the system's parameters, and so the proposed algorithm can be applied. The left-half plane is considered as the stability region $D$. Due to the symmetry, the contour of the region can be defined as $u(z)=j z, z \in \mathbf{Z}:=[0, \infty)$.

Because of the invariance of the coefficient of the highest order of the polynomial, it is immediately evident that $\eta=\infty$ from (10).

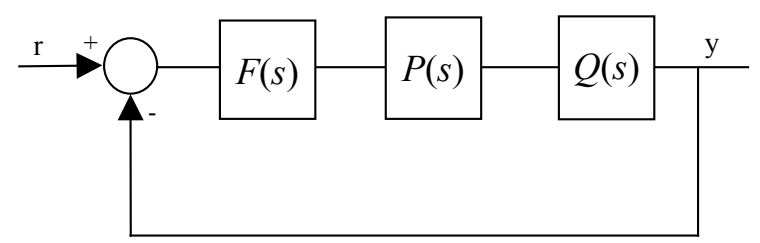

Fig. 1. Feedback control loop for the example.

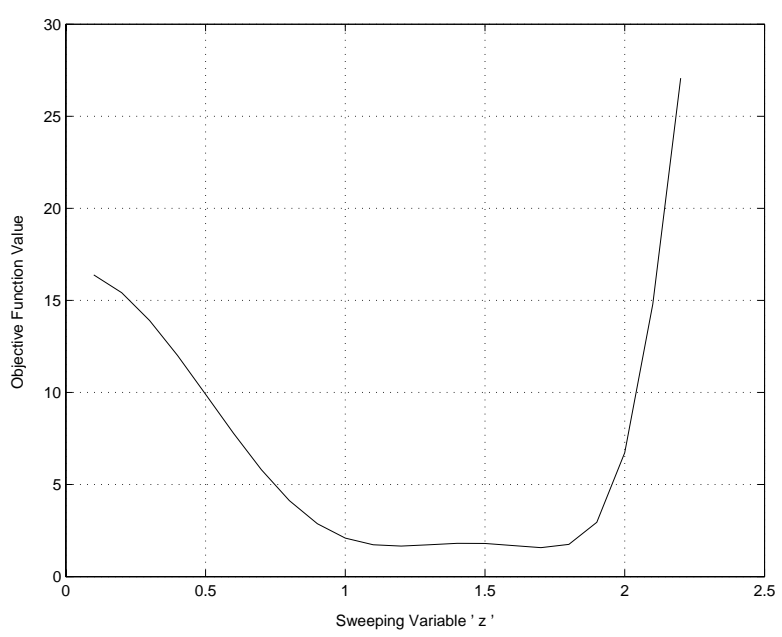

Fig. 2. The optimal values of the objective function of Problem MDE versus the sweeping variable.

Implementing the algorithm of Section 4 to solve the MDE problem, the optimal values of the objective function (13) are computed. This objective function is plotted in Fig. 2 for a selected range of the sweeping variable $z$. In the RLT, the $Q_{k}$-variables are defined as:

$$
\begin{aligned}
& Q_{1}, Q_{2}, Q_{3}, Q_{4}, Q_{\{1,1\}}, Q_{\{1,2\}}, Q_{\{1,3\}}, Q_{\{1,4\}}, \\
& Q_{\{2,2\}}, Q_{\{2,3\}}, Q_{\{2,4\}}, Q_{\{3,3\}}, Q_{\{3,4\}}, Q_{\{4,4\}} .
\end{aligned}
$$

The MDF $\rho(z)$ is the square root of the objective function. The minimum of $\rho(z)$ over $z \in \mathbf{Z}$ is obtained as $\rho\left(z^{*}\right)=(1.568)^{1 / 2}=1.252$ at $z^{*}=1.715$. The optimal value is obtained at $q=[3.391,0.844,5.281$, $2.029]$. Thus, from Theorem 2 , the stability margin is determined to be $\gamma=1.252$. To verify this result, we also solved (23) directly via the higher-order polynomial program as mentioned in Remark 3, and we obtained identical results. However, this run took a lot more CPU time. As a further validation, some $5 \times 10^{3}$ random points inside the hypersphere (8), centered at $q=\bar{q}$ and with radius of $b=1.252$ were considered by plotting the roots of the characteristic polynomial at these points (Fig. 3). It can be seen that the resultant root locus lies inside the desired region (left-half plane) and marginally touches the border of the region (the imaginary axis), which shows that the computed stability margin is "very tight".

\section{CONCLUSIONS}

In this paper, a method is presented for the computation of stability margins for LTI systems that have a polynomial uncertainty structure. An optimization problem is formulated for this purpose, and polynomial programming techniques are then used to solve the problem. The proposed approach optimally determines the size of the $D$-stability hypersphere around a nominal point in the parameter space, hence characterizing the $D$-stability margin. An example has been provided to illustrate the efficacy of this approach. 


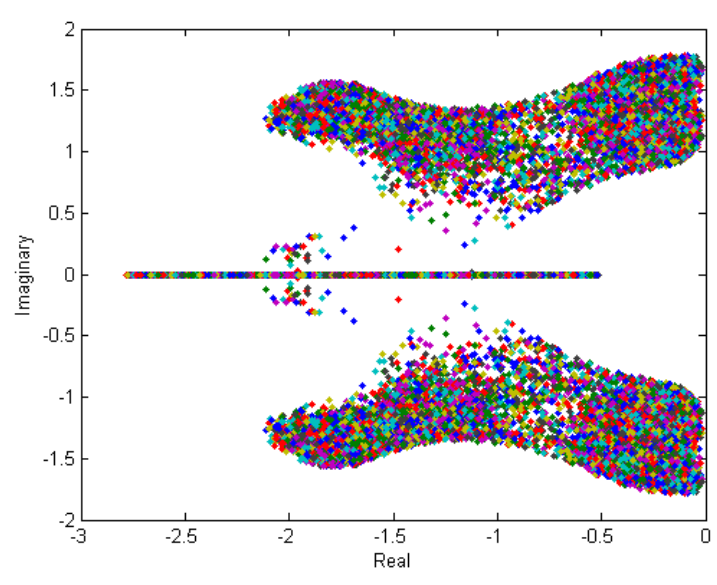

Fig.3. The root-locus of 5000 points inside the computed hypersphere showing that the perturbed closed loop system always remains stable, and that the bound obtained is tight.

Acknowledgements: The first author acknowledges the support of the "Research Council of University of Yazd" for supporting his visit to the Systems Control Group of University of Toronto, where this work was initiated. The work of the second author has been supported in part by the National Science Foundation under Grant Number 0094462. The authors also gratefully acknowledge the assistance of $\mathrm{Mr}$. Jitamitra Desai in helping with the computations for the numerical example.

\section{REFERENCES}

Ackermann, J. (2002). Robust Control: The Parameter Space Approach, Springer-Verlag, London, UK.

Ackermann, J., D. Kaesbauer and R. Muench (1990). Robust Gamma-stability analysis in a plant parameter space. Automatica, 27, 75-85.

Barmish, R.B. (1994). New Tools for Robustness of Linear Systems. Macmillan, New York, NY.

Bhattacharyya, S. P., H. Chapellat and L. H. Keel (1995). Robust Control: The Parametric Approach, Prentice-Hall, Englewood Cliffs, NJ.

Bozorg, M. and E. M. Nebot (1999). $l^{p}$ parameter perturbation and design of robust controllers for linear systems. International Journal of Control, 72, 267-275.

Chapellat, H., L. H. Keel and S. P. Bhattacharyya (1993). Robust stability manifolds for multilinear interval systems. IEEE Transactions on Automatic Control, 34, 314-318.

De Gaston, R. R. and M. G. Safonov (1988). Exact calculation of the multiloop stability margin. IEEE Transactions on Automatic Control, 33, 156-171.

Desages, A. C., L. Castro and H. Cendra (1991). Distance of a complex coefficient stable polynomial from the boundary of the stability set. Multidimensional Systems and Signal Processing, 2, 189-210.

Djaferis, T. E. (1995). Robust Control Design: A Polynomial Approach, Kluwer Academic Publishers, Norwell, MA.

Hinrichsen, D. and A. J. Pritchard (1989). An application of state space methods to obtain explicit formulae for robustness measures of polynomials. In: Robustness in Identification and Control, (M. Milanese, R. Tempo and A. Vicino. (Ed)), 125-206, Plenum Press, New York, NY.

Keel, L.H. and S. P. Bhattacharyya (1993). Parametric stability margin for multilinear interval control systems. Proceedings of American Control Conference, San Francisco, CA, 262-266.

Polyak, B. T. and J. Kogan (1995). Necessary and sufficient conditions for robust stability of linear systems with multiaffine uncertainty structure. IEEE Transactions on Automatic Control, 40, 1255-1260.

Qiu, L. and E. J. Davison (1989). A simple procedure for the exact stability robustness computation of polynomials with affine coefficient perturbations. Systems and Control Letters, 13, 413-420.

Rudin, W. (1976). Principles of Mathematical Analysis, Mc Graw-Hill, New York, NY.

Sherali, H. D. and C. H. Tuncbilek (1992). A global optimization algorithm for polynomial programming problems using a ReformulationLinearization Technique. Journal of Global Optimization, 2, 101-112.

Sherali, H. D. and C. H. Tuncbilek (1997). New Reformulation - Linearization Convexification relaxations for univariate and multivariate polynomial programming problems. Operations Research Letters, 21, 1-10.

Sideris, S. and R. S. Sánchez Peña (1989). Fast computation of multivariable stability margin for real interrelated uncertain parameters. IEEE Transactions on Automatic Control, 34, 12721276.

Tesi, A. and A. Vicino (1990). Robustness analysis for linear dynamical systems with linearly correlated parametric uncertainties. IEEE Transactions on Automatic Control, 35, 186-191.

Tsypkin, Y. Z. and B. T. Polyak (1991). Frequency domain criteria for robust stability of polytope of polynomials. In: Control of Uncertain Dynamic Systems, (S. P. Bhattacharyya and L. H. Keel (Ed)), 491-499, CRC Press, Boca Raton, FL.

Zadeh, L.A. and C. A. Desoer (1963). Linear Systems Theory, McGraw-Hill, New York, NY.

Zettler, M. and J. Garloff (1998). Robustness analysis of polynomials with polynomial parameter dependency using Bernstein expansion. IEEE Transactions on Automatic Control, 43, 425-431. 